\title{
Guest Editorial: Special Issue on Parameterized and Exact Computation, Part I
}

\author{
Venkatesh Raman • Saket Saurabh
}

Received: 1 December 2011 / Accepted: 12 December 2011 / Published online: 21 December 2011

(C) Springer Science+Business Media, LLC 2011

The papers in this issue form the first part of the two parts special issue in the area of parameterized and exact exponential algorithms and complexity. While some of these are selected and extended papers from International Symposium on Parameterized and Exact Computation (IPEC) 2010, the rest were selected from the submissions received for our call for the special issue in the area.

The two parts reflect the volume of high quality submissions we received, which in turn reflects the high growth of the area in recent times. The papers in this issue represent the subareas of recent growth in the area including variations of RobertsonSeymour and Courcelle's theorems, kernelization and structural parameterization.

In parameterized (or multivariate) complexity, the aim is to design and analyse algorithms focussing on various parameters in the input-besides the standard input size. In particular, for hard problems, the aim is to confine the combinatorial explosion of the running time of the algorithms to small parameters in the input. Besides giving rise to new and interesting algorithm design techniques and complexity paradigms, the area has been highly successful in domains (like computational biology and artificial intelligence) where small parameters govern practical situations.

While most of the initial work in the area considered the size of the solution as the parameter, there has been a lot of work in recent times to consider an ecology of parameters including various 'structural' parameters. The size of the vertex cover (which measures how far the graph is from an edgeless graph) and the feedback vertex set (which measures how far the graph is from a forest) are such parameters especially on graph problems. The paper 'Well Quasi orders in subclasses of bounded treewidth

V. Raman · S. Saurabh $(\bowtie)$

The Institute of Mathematical Sciences, Chennai, India

e-mail: saket@imsc.res.in

V. Raman

e-mail: vraman@imsc.res.in 
graphs and their algorithmic applications' revisits the classical Robertson-Seymour theorem in the classes of bounded treewidth graphs that have small vertex covers or feedback vertex sets or circumference (the size of the largest cycle). While we know that the class of all finite graphs are well quasi ordered under minor order, this paper asserts such a claim (and gives algorithmic applications) for several other orders, in these special classes of graphs. Similarly the paper 'Algorithmic Meta-Theorems for Restrictions of Treewidth' gives stronger algorithmic meta-theorems (similar to courcelle's theorem) giving better bounds for problems expressible in certain logic for graphs with bounded vertex cover number and bounded max-leaf number, subclasses of bounded treewidth graphs. The paper 'parameterized modal satisfiability' investigates the parameterized complexity of the satisfiability problem for modal logic using structural parameters (like modality depth) beyond the number of propositional variables. The paper 'A new lower bound on the maximum number of satisfied clauses in Max-SAT and its algorithmic applications' looks at MaxSAT where the parameter is the number of satisfiable clauses beyond some guaranteed lower bound. The authors first prove a new lower bound and give a fixed-parameter tractable algorithm for the problem.

The paper 'Fast minor testing for planar graphs' gives a fast (single exponential in the size of the host graph) algorithm to determine whether a host graph is a minor of a given planar graph.

The papers 'Parameterized complexity of spanning tree congestion problems', 'Parameterized complexity results for general factors and an application to constrained programming', and 'Multivariate complexity of swap bribery' investigate important specific problems using parameterized complexity under various input parameters relevant for the problems.

The papers 'Cluster editing: kernelization based on edge cuts' and 'An improved FPT algorithm and a quadratic kernel for pathwidth one vertex deletion' are in the recently emerging subareas of kernelization and deletion problems. The first one gives an improved kernel for the weighted version of the cluster editing problem (can we delete a subset of vertices of weight at most $k$ to obtain disjoint union of cliques) and the second one gives an improved fixed-parameter tractable algorithm and an improved kernel for the pathwidth one deletion problem (can we delete at most $k$ vertices to obtain a graph of pathwidth at most one). The paper 'Parameterized measure and conquer for problems with no small kernels' gives how the 'measure and conquer' paradigm can still be applied for obtaining improved exact and parameterized algorithms even for problems for which linear sized (or in some cases even polynomial sized) kernels are unlikely to exist, using two problems-Connected Vertex Cover and Edge dominating set—as examples.

All the papers in the volume went through the rigorous refereeing process of Algorithmica. We thank all those who submitted papers in response to our call for papers, and the referees for their time and effort. Finally, we thank Ming-Yang Kao, editorin-chief of Algorithmica for his guidance throughout the production of this special issue. 